\title{
NORMS OF POLYNOMIALS AND CAPACITIES ON BANACH SPACES
}

\author{
MIGUEL LACRUZ
}

AdDED IN PROOF. Although I never submitted this paper for publication anywhere else, by a mistake I sent it to Integral Equations Operator Theory who printed it, also by a mistake, in Vol. 34 (4) (1999), 494-499.

\section{Introduction}

Recall that a function $P$ defined on a complex Banach space $X$ is a homogeneous polynomial of degree 0 if $P$ is constant, a homogeneous polynomial of degree $d \geq 1$ if there is a bounded, symmetric $d$-linear form $A$ on $X$ such that $P(x)=A(x, \ldots, x)$ for all $x \in X$, and a polynomial of degree $d$ if $P$ can be written as $P=P_{0}+P_{1}+\cdots+P_{d}$, where each $P_{k}$ is a homogeneous polynomial of degree $k$. There is a natural norm on the vector space of polynomials; it is given by the expression

$$
\|P\|=\sup \left\{|P(x)|: x \in B_{X}\right\} .
$$

This paper concerns a general procedure for estimating norms of polynomials. Such procedure can be described as follows. Let $S$ be a subset of $B_{X}$, put

$$
\begin{aligned}
|P|_{S} & =\sup \{|P(x)|: x \in S\}, \\
c_{d}(S) & =\sup \left\{\|P\|: \operatorname{deg} P=d,|P|_{S} \leq 1\right\},
\end{aligned}
$$

and observe that the inequality $|P|_{S} \leq\|P\| \leq c_{d}(S)|P|_{S}$ holds for every polynomial $P$ of degree $d$ on $X$. Thus, giving upper bounds on $c_{d}(S)$ provides a way for estimating $\|P\|$ in terms of $|P|_{S}$.

Received February 5, 1997. The paper has appeared in Integral Equations Operator Theory 34 (4) (1999), 494-499 because of the following course of events. The author submitted a different paper to IEOT February 16, 1998. This was accepted March 3, 1998. By a mistake the author sent the manuscript of the wrong paper to IEOT October 22, 1998, believing it to be the proof of the paper he submitted to IEOT. IEOT did not discover that they had received the wrong paper, and the wrong paper was printed and published July 30, 1999. Math. Scand. was informed about the wrong publication November 22, 1999, too late to make large changes in the page proof of vol 85.2. 
Durand [2] introduced this scheme for polynomials in one complex variable and computed the precise values of $c_{d}(S)$ for some specific subsets of the unit disc, obtaining the results that are shown in the table below.

\begin{tabular}{|c|c|}
\hline$S$ & $c_{d}(S)$ \\
\hline$\{z \in C:|z| \leq r\}$ & $r^{-d}$ \\
\hline$\left\{e^{i t} \in C:|t-\theta| \leq \frac{\varepsilon}{2}\right\}$ & $\frac{1}{2}\left[\left(\tan \frac{\varepsilon}{8}\right)^{d}+\left(\tan \frac{\varepsilon}{8}\right)^{-d}\right]$ \\
\hline$[0,1]$ & $\frac{1}{2}\left[(3+\sqrt{2})^{d}+(3-2 \sqrt{2})^{d}\right]$ \\
\hline$\left\{z \in C: z^{d k}=1\right\}, k \geq 2$ & {$\left[\sin \frac{\pi(k-1)}{2 k}\right]^{-1}$} \\
\hline
\end{tabular}

Aron, Beauzamy, and Enflo [1] analyzed the problem of comparing real and complex norms of polynomials in many variables. They looked at polynomials $P$ of degree $d$ in $N$ variables

$$
P\left(x_{1}, \ldots, x_{N}\right)=\sum_{|\alpha| \leq d} a_{\alpha} x_{1}^{\alpha_{1}} \cdots x_{N}^{\alpha-N}
$$

and considered the norms

$$
\begin{aligned}
& \|P\|_{R}=\sup \left\{\left|P\left(x_{1}, \ldots, x_{N}\right)\right|:-1 \leq x_{1}, \ldots, x_{N} \leq 1\right\}, \\
& \|P\|_{C}=\sup \left\{\left|P\left(e^{i t_{1}}, \ldots, e^{i t_{N}}\right)\right|: 0 \leq t_{1}, \ldots, t_{N} \leq 2 \pi\right\},
\end{aligned}
$$

for which they obtained the inequality

$$
\|P\|_{C} \leq \frac{(3 \sqrt{2}+4)^{d}+(3 \sqrt{2}-4)^{d}}{2}\|P\|_{R}
$$

This estimate represents an upper bound on $c_{d}(S)$ when $X=\ell_{\infty}^{(N)}$ and $S=[-1,1]^{N}$. Notice that the bound is independent of the number of variables.

Siciak [6] improved their inequality later on. Using the notions of extremal functions and capacities in $C^{N}$ allowed him to obtain the sharp estimate

$$
c_{d}\left([-1,1]^{N}\right)=(1+\sqrt{2})^{d} .
$$

The aim of this paper is on the one hand to give upper bounds on $c_{d}(S)$ for certain subsets of general Banach spaces, and on the other hand to extend Durand's inequalities to polynomials in many variables. 


\section{Results on general Banach spaces}

This section is devoted to give an upper estimate on $c_{d}(S)$ when $S$ is an $\varepsilon$-net of $B_{X}$ and to compute $c_{d}(S)$ when $S$ is a ball of radius $r>0$ centered at $a \in X$.

Theorem 2.1. Let $X$ be a complex Banach space, let $d \geq 1$, and let $0<\varepsilon<\log 2 /(e d)$. If $S_{\varepsilon}$ is an $\varepsilon-n e t$ of $B_{X}$ then

$$
c_{d}\left(S_{\varepsilon}\right) \leq \frac{1}{2-e^{e d \varepsilon}} .
$$

The proof of Theorem 2.1 relies on an infinite-dimensional version of Bernstein's inequality due to Harris [3], who stated it only for homogeneous polynomials, although it also works for arbitrary polynomials, as Tonge and Lacruz [4] pointed out.

Lemma 2.2 (Harris-Bernstein inequality). Let $P$ be a polynomial of degree $d$ on $X$ and let $D^{k} P$ denote its $k$ th Fréchet derivative, where $1 \leq k \leq d$. If $x, y \in B_{X}$ then

$$
\left|D^{k} P(x)\left(y^{k}\right)\right| \leq \frac{d^{d}}{(d-k)^{d-k}}\|P\| .
$$

Proof of Theorem 2.1. Let $P$ be a polynomial of degree $d$ on $X$, let $\eta>0$, and pick an $x \in B_{X}$ with $|P(x)| \geq(1-\eta)\|P\|$. Now take a point $y \in S_{\varepsilon}$ such that $\|x-y\|<\varepsilon$ and expand $P$ in Taylor series around $y$ to obtain

$$
P(x)-P(y)=\sum_{k=1}^{d} \frac{\|x-y\|^{k}}{k !} D^{k} P(y)\left(\frac{x-y}{\|x-y\|}\right)^{k} .
$$

It follows from Harris-Bernstein inequality that

$$
\left.|P(x)-P(y)| \leq\|P\| \sum_{k=1}^{d} \frac{d^{d}}{(d-k)^{d-k}} \frac{\varepsilon^{k}}{k !},\right)
$$

but $[d /(d-k)]^{d-k} \leq e^{k}$ so the last expression is

$$
\leq\|P\| \sum_{k=1}^{d} \frac{(e d \varepsilon)^{k}}{k !} \leq\|P\|\left(e^{e d \varepsilon}-1\right) .
$$

Hence, $(1-\eta)\|P\| \leq|P(x)-P(y)|+|P(y)| \leq\|P\|\left(e^{e d \varepsilon}-1\right)+|P(y)|$, which leads to the inequality $|P(y)| \geq\|P\|\left(2-e^{e d \varepsilon}-\eta\right)$. Since $\eta>0$ is arbitrary, it follows that $|P|_{S_{\varepsilon}} \geq\|P\|\left(2-e^{e d \varepsilon}\right)$. 
Remarks. (i) Notice that $c_{d}\left(S_{\varepsilon}\right) \rightarrow 1$ as $\varepsilon \rightarrow 0+$, so that the error when approximating $\|P\|$ in terms of $|P|_{S}$ can be made as small as desired.

(ii) Let $T$ be a subset of $B_{X}$ such that $\|P\|=|P|_{T}$ for every $P$. The proof of Theorem 2.1 shows that the same result holds when $S_{\varepsilon}$ is just an $\varepsilon$-net of $T$. This is the case when $X=\ell_{\infty}^{(N)}, T$ is the distinguished boundary of the polydisc, and $S_{\varepsilon}$ consists of the points in $\mathrm{C}^{N}$ all of whose coordinates are $d k$ th roots of unity. This can be regarded as an extension of Durand's fourth result.

TheORem 2.3. Let $X$ be a complex Banach space, let $a \in X$, and let $r>0$. If $d \geq 1$ then

$$
c_{d}\left(a+r B_{X}\right) \leq\left(\frac{1+\|a\|}{r}\right)^{d},
$$

and the estimate is best possible.

Proof of Theorem 2.3. First of all consider the case $a=0$. Let $\eta>0$, pick an $x \in B_{X}$ such that $|P(x)| \geq(1-\eta)\|P\|$, and define $f(z)=P(z x)$. Then $f(z)$ is a polynomial of degree $d$ in one complex variable. Now apply Durand's first result to get

$$
\frac{1}{r^{d}} \sup \{|f(z)|:|z| \leq r\} \geq\|f\| \geq|f(1)|=|P(x)| \geq(1-\eta)\|P\|
$$

so that there exists a $z \in C$ with $|z| \leq r$ and $|P(z x)| \geq(1-\eta) r^{d}\|P\|$. This gives the desired inequality, since $\|z x\| \leq r$ and $\eta>0$ is arbitrary.

Next, no longer assume $a=0$, consider the polynomial $Q(x)=P(x-a)$ and observe that

$$
\begin{aligned}
\frac{1}{r^{d}}|P|_{a+r B_{X}} & =\frac{1}{r^{d}}|Q|_{r B_{X}} \geq\|Q\|=\sup \left\{|Q((1+\|a\|) x)|:\|x\| \leq \frac{1}{1+\|a\|}\right\} \\
& \geq \frac{1}{(1+\|a\|)} \sup \{|Q((1+\|a\|) x)|:\|x\| \leq 1\} \\
& =\frac{1}{(1+\|a\|)} \sup \{|Q(y)|:\|y\| \leq 1+\|a\|\} \\
& \geq \frac{1}{(1+\|a\|)} \sup \{|Q(x+a)|:\|x\| \leq 1\}=\frac{1}{(1+\|a\|) d}\|P\|,
\end{aligned}
$$

so the inequality follows. Finally, consider the polynomial $f(z)=$ $[(z-a) / r]^{d}$, where $a \in \mathrm{C}$ and $r>0$, and conclude that the inequality is best possible. 


\section{Extremal functions and capacities}

According to Siciak [5], the extremal function associated with a subset $S \subseteq C^{N}$ is defined by

$$
\Phi_{S}(x)=\sup _{d \geq 1} \sqrt[d]{\Phi_{d}(x)},
$$

where $\Phi_{d}(x)=\sup \left\{|P(x)|: \operatorname{deg} P=d,|P|_{S}=1\right\}$. The $\alpha$-capacity of $S$ is defined by

$$
\frac{1}{\alpha(S)}=\sup \left\{\Phi_{S}(x):\|x\| \leq 1\right\} .
$$

Notice that these definitions also make sense when $S$ is a subset of a complex Banach space $X$. The following result is a well-known fact about sequences of real numbers.

Lemma 3.1. Let $\left\{a_{d}\right\}_{d=1}^{\infty}$ be a sequence of positive numbers that satisfies the condition $a_{d+d^{\prime}} \geq a_{d} a_{d^{\prime}}$ for all $d, d^{\prime} \geq 1$. Then

$$
\lim _{d \rightarrow \infty} \sqrt[d]{a_{d}}=\sup _{d \geq 1} a_{d} .
$$

Since the product of a polynomial of degree $d$ and a polynomial of degree $d^{\prime}$ is a polynomial of degree $d+d^{\prime}$, it follows from Lemma 3.1 that

$$
\Phi_{S}(x)=\lim _{d \rightarrow \infty} \sqrt[d]{\Phi_{d}(x)} .
$$

There is a strong connection between the $\alpha$-capacity of a set $S$ and the quantities $c_{d}(S)$; it is given by the following

Lemma 3.2. If $S$ is a subset of a complex Banach space then

$$
\frac{1}{\alpha(S)}=\sup _{d \geq 1} \sqrt[d]{c_{d}(S)}=\lim _{d \rightarrow \infty} \sqrt[d]{c_{d}(S)}
$$

Proof of Lemma 3.2. First of all, notice that

$$
\begin{aligned}
\frac{1}{\alpha(S)} & =\sup _{x \in B_{X}} \Phi_{S}(x)=\sup _{x \in B_{X}} \sup _{d \geq 1} \sqrt[d]{\Phi_{d}(x)} \\
& =\sup _{d \geq 1} \sup _{x \in B_{X}} \sqrt[d]{\Phi_{d}(x)}=\sup _{d \geq 1} \sqrt[d]{c_{d}(S)} .
\end{aligned}
$$

The second equality follows from Lemma 3.1, taking into account the submultiplicativity of the norm of polynomials. 
Notice that, as an application of Lemma 3.2, the result obtained in Theorem 2.3 admits of an equivalent formulation in terms of capacities, namely,

$$
\alpha\left(a+r B_{X}\right)=\frac{r}{1+\|a\|} .
$$

\section{Estimates for polynomials in many variables}

The following result is due to Siciak [5]; it concerns the computation of the maximal function for a product of subsets of the complex plane.

Lemma 4.1. Let $S_{1}, \ldots, S_{N} \subseteq \mathrm{C}$ and let $S=S_{1} \times \cdots \times S_{N} \subseteq \mathrm{C}^{N}$. If $z=\left(z_{1}, \ldots, z_{N}\right) \in \mathrm{C}^{N}$ then

$$
\Phi_{S}(z)=\max \left\{\Phi_{S_{1}}\left(z_{1}\right), \ldots, \Phi_{S_{N}}\left(z_{N}\right)\right\} .
$$

A straightforward consequence of Lemma 4.1 is the following

Lemma 4.2. Suppose $S_{1}, \ldots, S_{N} \subseteq \mathrm{C}$ and let $S=S_{1} \times \cdots \times S_{N} \subseteq \ell_{\infty}^{(N)}$. Then

$$
\alpha(S)=\min \left\{\alpha\left(S_{1}\right), \ldots, \alpha\left(S_{N}\right)\right\} .
$$

Proof of Lemma 4.2. Just notice that

$$
\begin{aligned}
\frac{1}{\alpha(S)} & =\sup _{\|z\|_{\infty} \leq 1} \Phi_{S}(z)=\sup _{\left|z_{k}\right| \leq 1} \max \left\{\Phi_{S_{1}}\left(z_{1}\right), \ldots, \Phi_{S_{N}}\left(z_{N}\right)\right\} \\
& =\max \left\{\sup _{\left|z_{1}\right| \leq 1} \Phi_{S_{1}}\left(z_{1}\right), \ldots, \sup _{\left|z_{N}\right| \leq 1} \Phi_{S_{N}}\left(z_{N}\right)\right\}=\max \left\{\frac{1}{\alpha\left(S_{1}\right)}, \ldots, \frac{1}{\alpha\left(S_{N}\right)}\right\},
\end{aligned}
$$

and the desired identity follows at once.

Now everything is ready to extend Durand's second result to polynomials in many variables.

TheOrem 4.3. Let $X=\ell_{\infty}^{(N)}$ and $\Gamma=\left\{\left(e^{i t_{1}}, \ldots, e^{i t_{N}}\right) \in \mathrm{C}^{N}:\left|t_{k}-\theta_{k}\right| \leq\right.$ $\left.\varepsilon_{k} / 2\right\}$, where $\theta_{1}, \ldots, \theta_{N}$ are fixed real numbers and $0<\varepsilon_{1}, \ldots, \varepsilon_{N}<2 \pi$. Then

$$
c_{d}(\Gamma) \leq \cot \left(\frac{\varepsilon}{8}\right)^{d},
$$

where $\varepsilon=\min \left\{\varepsilon_{1}, \ldots, \varepsilon_{N}\right\}$.

Proof of Theorem 4.3. For each $1 \leq k \leq N$, put $\Gamma_{k}=\left\{\left(e^{i t_{k}} \in \mathrm{C}\right.\right.$ : $\left.\left|t_{k}-\theta_{k}\right| \leq \varepsilon_{k} / 2\right\}$, so that $\Gamma=\Gamma_{1} \times \cdots \times \Gamma_{N}$. Durand's second result in combination with Lemma 3.2 leads to 


$$
\begin{aligned}
\frac{1}{\alpha\left(\Gamma_{k}\right)} & =\lim _{d \rightarrow \infty} \sqrt[d]{c_{d}\left(\Gamma_{k}\right)} \\
& =\lim _{d \rightarrow \infty} \frac{c_{d+1}\left(\Gamma_{k}\right)}{c_{d}\left(\Gamma_{k}\right)}=\cot \left(\frac{\varepsilon_{k}}{8}\right) .
\end{aligned}
$$

Now Lemma 4.2 gives

$$
\begin{aligned}
\alpha(\Gamma) & =\min \left\{\alpha\left(\Gamma_{1}\right), \ldots, \alpha\left(\Gamma_{N}\right)\right\} \\
& =\min \left\{\tan \left(\frac{\varepsilon_{1}}{8}\right), \ldots, \tan \left(\frac{\varepsilon_{N}}{8}\right)\right\}=\tan \left(\frac{\varepsilon}{8}\right) .
\end{aligned}
$$

Thus, $c_{d}(\Gamma) \leq 1 / \alpha(\Gamma)^{d}=\cot \left(\frac{\varepsilon}{8}\right)^{d}$.

\section{REFERENCES}

1. R. Aron, B. Beauzamy, and P. Enflo, Polynomials in many variables: real vs. complex norms, J. Approx. Theory 74, no. 2, 1993.

2. A. Durand, Quelques aspects de la théorie analytique des polynômes, Lecture Notes in Math. $1415,1990$.

3. L. A. Harris, Bounds on the derivatives of holomorphic functions of vectors, Colloque d'Analyse, ed. L. Nachbin, Rio de Janeiro, 1972.

4. M. Lacruz and A. M. Tonge, Polynomials on Banach spaces: zeros and maximal points, J. Math. Anal. Appl. 192, no. 2, 1995.

5. J. Siciak, On some extremal functions and their applications in the theory of several complex variables, Trans. Amer. Math. Soc. 105, no. 2, 1962.

6. J. Siciak, Wiener's type sufficient conditions in $\mathrm{C}^{N}$, preprint.

DEPARTAMENTO DE ANÁLISIS MATEMÁTICO

FACULTAD DE CIENCIAS MATEMÁTICAS

UNIVERSIDAD DE SEVILLA

APARTADO DE CORREOS 1160

41080 SEVILLA (SPAIN)

lacruz@cica.es 